\title{
Phylogenetic analysis and classification of the Brassica rapa SET-domain protein family
}

Yong Huang ${ }^{1,2}$, Chunlin Liu', Wen-Hui Shen ${ }^{3 *}$ and Ying Ruan ${ }^{1,2^{*}}$

\begin{abstract}
Background: The SET (Su(var)3-9, Enhancer-of-zeste, Trithorax) domain is an evolutionarily conserved sequence of approximately 130-150 amino acids, and constitutes the catalytic site of lysine methyltransferases (KMTs). KMTs perform many crucial biological functions via histone methylation of chromatin. Histone methylation marks are interpreted differently depending on the histone type (i.e. H3 or H4), the lysine position (e.g. H3K4, H3K9, H3K27, H3K36 or H4K20) and the number of added methyl groups (i.e. me1, me2 or me3). For example, H3K4me3 and H3K36me3 are associated with transcriptional activation, but H3K9me2 and H3K27me3 are associated with gene silencing. The substrate specificity and activity of KMTs are determined by sequences within the SET domain and other regions of the protein.

Results: Here we identified 49 SET-domain proteins from the recently sequenced Brassica rapa genome. We performed sequence similarity and protein domain organization analysis of these proteins, along with the SETdomain proteins from the dicot Arabidopsis thaliana, the monocots Oryza sativa and Brachypodium distachyon, and the green alga Ostreococcus tauri. We showed that plant SET-domain proteins can be grouped into 6 distinct classes, namely KMT1, KMT2, KMT3, KMT6, KMT7 and S-ET. Apart from the S-ET class, which has an interrupted SET domain and may be involved in methylation of nonhistone proteins, the other classes have characteristics of histone methyltransferases exhibiting different substrate specificities: KMT1 for H3K9, KMT2 for H3K4, KMT3 for H3K36, KMT6 for H3K27 and KMT7 also for H3K4. We also propose a coherent and rational nomenclature for plant SET-domain proteins. Comparisons of sequence similarity and synteny of B. rapa and A. thaliana SET-domain proteins revealed recent gene duplication events for some KMTs.

Conclusion: This study provides the first characterization of the SET-domain KMT proteins of B. rapa. Phylogenetic analysis data allowed the development of a coherent and rational nomenclature of this important family of proteins in plants, as in animals. The results obtained in this study will provide a base for nomenclature of KMTs in other plant species and facilitate the functional characterization of these important epigenetic regulatory genes in Brassica crops.
\end{abstract}

Keywords: Chromatin, Histone, Lysine methylation, SET domain, Gene duplication, Nomenclature

\section{Background}

Epigenetic regulation acts through heritable changes in genome function that occur without a change in DNA sequence. One well-known epigenetic mechanism is through posttranslational covalent modifications of histones; these modifications include acetylation, methylation,

\footnotetext{
* Correspondence: wen-hui.shen@ibmp-cnrs.unistra.fr; yingruan@hotmail.com ${ }^{1}$ Hunan Provincial Key Laboratory of Crop Germplasm Innovation and Utilization, Hunan Agricultural University, Changsha 410128, China ${ }^{3}$ Institut de Biologie Moléculaire des Plantes du CNRS, Université de Strasbourg, 12 rue du Général Zimmer, 67084 Strasbourg Cedex, France Full list of author information is available at the end of the article
}

ubiquitylation and others, and form the basis of the 'histone code' for gene regulation [1]. Histone lysine methylation plays a pivotal role in a wide range of cellular processes including heterochromatin formation, transcriptional regulation, parental imprinting, and cell fate determination [2]. At least six lysine residues, five on histone H3 (K4, K9, K27, K36, K79) and one on H4 (K20), are subject to methylation. Each lysine can carry one, two or three methyl residue(s), known as mono-, di- and tri-methylation, respectively. In general, di-/tri- methylation of H3K4 and H3K36 correlates with transcriptional activation, whereas di-methylation of $\mathrm{H} 3 \mathrm{~K} 9$ and trimethylation of

\section{Biomed Central}


H3K27 correlates with gene silencing in plants and animals $[2,3]$.

All known lysine methylation modifications, with the exception of H3K79 methylation, are carried out by methyltransferases that contain an evolutionarily conserved SET domain, named after three Drosophila genes (Su(var), E(z), and Trithorax) [4]. The SET domain encompasses approximately 130-150 amino acids that form a knot-like structure and constitute the enzyme catalytic site for lysine methylation [5]. In addition to the SET domain, flanking sequences, more distant protein domains, and possibly some cofactors are also important for enzyme activity and specificity. The genes encoding SET-domain proteins are ancient, existing in prokaryotes and eukaryotes, but have proliferated and evolved novel functions connected with the appearance of eukaryotes [6].

The first plant genes encoding SET-domain proteins to be genetically characterized were CURLY LEAF (CLF) and MEDEA (MEA) in Arabidopsis thaliana [7,8]. Chromatinbinding properties and histone methylation activity of plant SET-domain proteins were first reported for tobacco NtSET1 and Arabidopsis KRYPTONITE (KYP) $[9,10]$. Phylogenetic analysis of plant SET domain proteins has proven helpful as a guide for genetic and molecular studies of this large family of proteins $[11,12]$. To date, some of the Arabidopsis SET-domain family members have been characterized and shown to play crucial functions in diverse processes including flowering time control, cell fate determination, leaf morphogenesis, floral organogenesis, parental imprinting and seed development [3,13-15].

Genome sequences of an increasing number of plant species, in addition to the model plants (Arabidopsis thaliana, Oryza sativa, and Brachypodium distachyon), have also been completed. Other Brassica species are of particular interest because of their agro-economical importance and their close relationship with Arabidopsis, thus providing insights into recent SET-domain gene amplification during evolution of Brassica species. Here, we identified and analyzed 49 SET-domain proteins from the recently completed Brassica rapa whole genome sequence [16]. Our data provide a platform for future functional characterization of these important epigenetic regulatory genes in Brassica species.

\section{Results \\ Identification of SET-domain proteins from the B. rapa genome}

Using BLASTp and tBLASTn with the full complement of known Arabidopsis and rice SET-domain proteins as queries, we identified 49 genes encoding different SET-domain proteins from the B. rapa genome (http:// brassicadb.org/brad). We used the nomenclature recently proposed for lysine methyltransferases (KMTs,
[17]) and named the newly identified $B$. rapa genes based on our phylogenetic analysis of their corresponding protein sequences (see below). Apart from $B r K M T 1 B ; 1 a$ and BrKMT1B;2b genes, whose chromosomal locations are yet unknown, the other 47 genes are distributed on the ten $B$. rapa chromosomes, with 1-7 KMT genes per chromosome (Table 1).

\section{B. rapa SET-domain proteins can be grouped into six classes}

To analyze the $B$. rapa SET-domain protein sequences, we extracted SET-domain proteins from several other green lineage species, including 37 proteins from A. thaliana, 36 proteins from $O$. sativa, 41 proteins from $B$. distachyon, and 10 proteins from Ostreococcus tauri (Table 1). We also included the Saccharomyces cerevisiae ScKMT2/Set1 and ScKMT3/Set2 proteins, which are H3K4- and H3K36specific KMTs, respectively [18,19], and can be used to represent ancient eukaryotic SET-domain proteins from an evolutionary point of view. Phylogenetic analysis of the aforementioned 175 SET-domain proteins revealed that they could be grouped into 6 distinct classes, namely KMT1, KMT2, KMT3, KMT6, KMT7 and S-ET class (Figure 1). The first four class numbers used here are consistent with the nomenclature previously proposed for yeast and animal KMTs [17]. Furthermore, two plant-specific subclasses (namely A and B) were identified for KMT1 and KMT6. Representative members of each class/ subclass are found in A. thaliana, B. rapa, O. sativa and $B$. distachyon. The S-ET class members contain an interrupted SET domain and are likely involved in methylation of nonhistone proteins, e.g. RUBISCO subunits; however, their biological functions remain largely unknown. Hereafter, we focused on the KMT classes/subclasses that are involved in histone methylation.

\section{The KMT1A subclass proteins}

The KMT1A subclass is the largest class and can be further divided into 4distinct groups (Figure 2). In each group, proteins from dicots (B. rapa and A. thaliana) and monocots (O. sativa and $B$. distachyon) clearly fall into separate branches, indicating that they are derived from a common ancestral gene but diverged before the monocot/dicot separation. The first three groups have a relatively simple relationship and small number of genes, but Group-4 is more complex: each plant species has 4-8 members that diverged at various times during evolution. In the case of B. rapa, among the 8 members belonging to Group-4, BrKMT1A;4a, BrKMT1A;4c, BrKMT1A;4d, and BrKMT1A;4f are clustered with the Arabidopsis AtKMT1A;4a/SDG32/SUVH1; BrKMT1A;4b with AtKMT1A;4b/SDG19/SUVH3; and BrKMT1A;4e, BrKMT1A;4 g and BrKMT1A;4 h with AtKMT1A;4e/SDG11/SUVH10 (Figure 2). Examination of synteny between $B$. rapa and $A$. 
Table 1 List of green lingeage SET-domain proteins analyzed in this study

\begin{tabular}{|c|c|c|c|c|c|c|c|c|c|c|}
\hline \multirow{2}{*}{$\begin{array}{l}\text { B. rapa } \\
\text { Name }\end{array}$} & \multirow[b]{2}{*}{ Locus } & \multirow[b]{2}{*}{ Chr. } & \multicolumn{2}{|l|}{ A. thaliana } & \multicolumn{2}{|l|}{ O. sativa } & \multicolumn{2}{|l|}{ B. distachyon } & \multicolumn{2}{|l|}{ O. tauri } \\
\hline & & & Name & Synonyms & Name & Synonyms & Name & Locus & Name & Synonyms \\
\hline BrKMT1A;1 & Bra006226 & 3 & AtKMT1A;1 & SDG33/SUVH4/KYP & OsKMT1A;1 & SDG714 & BdKMT1A;1a & Bradi2g59430 & & \\
\hline BrKMT1A;2a & Bra021840 & 4 & AtKMT1A;2a & SDG3/SUVH2 & OsKMT1A;2a & SDG726 & BdKMT1A;1b & Bradi2g26840 & & \\
\hline BrKMT1A;2b & Bra007048 & 3 & AtKMT1A;2b & SDG22/SUVH9 & OsKMT1A;2b & SDG715 & BdKMT1A;2a & Bradi1g29010 & & \\
\hline BrKMT1A;2C & Bra005511 & 5 & AtKMT1A;3a & SDG9/SUVH5 & OsKMT1A;3a & SDG710 & BdKMT1A;2b & Bradi3g12710 & & \\
\hline BrKMT1A;3a & Bra005362 & 5 & AtKMT1A;3b & SDG23/SUVH6 & OsKMT1A;3b & SDG727 & BdKMT1A;2C & Bradi2g60080 & & \\
\hline BrKMT1A;3b & Bra030212 & 4 & AtKMT1A;4a & SDG32/SUVH1 & OsKMT1A;3c & SDG703 & BdKMT1A;3a & Bradi3g35330 & & \\
\hline BrKMT1A;4a & Bra009408 & 10 & AtKMT1A;4b & SDG19/SUVH3 & OsKMT1A;4a & SDG704 & BdKMT1A;3b & Bradi4g28460 & & \\
\hline BrKMT1A;4b & Bra016018 & 7 & AtKMT1A;4C & SDG17/SUVH7 & OsKMT1A;4b & SDG713 & BdKMT1A;3C & Bradi4g28792 & & \\
\hline BrKMT1A;4c & Bra028776 & 2 & AtKMT1A;4d & SDG21/SUVH8 & OsKMT1A;4c & SDG728 & BdKMT1A;3d & Bradi5g17340 & & \\
\hline BrKMT1A;4d & Bra005829 & 3 & AtKMT1A;4e & SDG11/SUVH10 & OsKMT1A;4d & SDG709 & BdKMT1A;4a & Bradi1g53840 & & \\
\hline BrKMT1A;4e & Bra004258 & 7 & & & OsKMT1A;4e & SDG734 & BdKMT1A;4b & Bradi4g25940 & & \\
\hline BrKMT1A;4f & Bra025949 & 6 & & & OsKMT1A;4f & SDG733 & BdKMT1A;4C & Bradi2g13416 & & \\
\hline BrKMT1A;4g & Bra009409 & 10 & & & & & BdKMT1A;4d & Bradi4g43717 & & \\
\hline BrKMT1A;4h & Bra025950 & 6 & & & & & & & & \\
\hline BrKMT1B;1a & Bra040197 & $u d^{*}$ & AtKMT1B;1 & SDG31/SUVR4 & OsKMT1B;1 & SDG742 & BdKMT1B;1a & Bradi3g12790 & & \\
\hline BrKMT1B;1b & Bra001104 & 3 & AtKMT1B;2a & SDG13/SUVR1 & OsKMT1B;2 & SDG712 & BdKMT1B;1b & Bradi5g00500 & & \\
\hline BrKMT1B;2a & Bra033710 & 6 & AtKMT1B;2b & SDG18/SUVR2 & OsKMT1B;3 & SDG706 & BdKMT1B;2a & Bradi1g64870 & & \\
\hline BrKMT1B;2b & Bra036004 & $\mathrm{ud}^{*}$ & AtKMT1B;3 & SDG6/SUVR5/AtCZS & OsKMT1B;4 & SDG729 & BdKMT1B;2b & Bradi3g48970 & & \\
\hline BrKMT1B;3 & Bra032148 & 4 & AtKMT1B;4 & SDG20/SUVR3 & & & BdKMT1B;2C & Bradi3g48980 & & \\
\hline \multirow[t]{2}{*}{ BrKMT1B;4 } & Bra031976 & 2 & & & & & BdKMT1B;3 & Bradi3g52950 & & \\
\hline & & & & & & & BdKMT1B;4 & Bradi2g51320 & & \\
\hline BrKMT2;1a & Bra021721 & 4 & AtKMT2;1a & SDG27/ATX1 & OsKMT2;1 & SDG723 & BdKMT2;1 & Bradi4g08510 & OtKMT2 & SDG3601 \\
\hline BrKMT2;1b & Bra032450 & 9 & AtKMT2;1b & SDG30/ATX2 & OsKMT2;2a & SDG717 & BdKMT2;2a & Bradi4g01790 & & \\
\hline BrKMT2;2 & Bra027983 & 9 & AtKMT2;2 & SDG25/ATXR7 & OsKMT2;2b & SDG732 & BdKMT2;2b & Bradi3g13430 & & \\
\hline BrKMT2;3a & Bra003467 & 7 & AtKMT2;3a & SDG14/ATX3 & OsKMT2;3a & SDG721 & BdKMT2;2C & Bradi4g37627 & & \\
\hline BrKMT2;3b & Bra040838 & 8 & AtKMT2;3b & SDG16/ATX4 & OsKMT2;3b & SDG705 & BdKMT2;3a & Bradi2g07082 & & \\
\hline BrKMT2;3c & Bra003056 & 10 & AtKMT2;3c & SDG29/ATX5 & & & BdKMT2;3b & Bradi2g45430 & & \\
\hline BrKMT3;1 & Bra015678 & 7 & AtKMT3;1 & $\begin{array}{l}\text { SDG8/ASHH2/EFS/ } \\
\text { CCR1 }\end{array}$ & OsKMT3;1 & SDG725 & BdKMT3;1 & Bradi3g45727 & OtKMT3;1 & SDG3605 \\
\hline BrKMT3;2 & Bra015723 & 7 & AtKMT3;2 & SDG26ASHH1 & OsKMT3;2 & SDG708 & BdKMT3;2 & Bradi5g10110 & OtKMT3;2 & SDG3608 \\
\hline BrKMT3;3 & Bra010270 & 8 & AtKMT3;3 & SDG4/ASHR3 & OsKMT3;3a & SDG736 & BdKMT3;3a & Bradi3g48497 & & \\
\hline BrKMT3;4a & Bra004809 & 5 & AtKMT3;4a & $\mathrm{SDG} 7 / \mathrm{ASHH} 3$ & OsKMT3;3b & SDG707 & BdKMT3;3b & Bradi3g36790 & & \\
\hline BrKMT3;4b & Bra00396 & 7 & AtKMT3;4b & SDG24/ASHH4 & OsKMT3;4 & SDG724 & BdKMT3;4a & Bradi1g20527 & & \\
\hline BrKMT3;4c & Bra00343 & 3 & & & & & BdKMT3;4b & Bradi4g27797 & & \\
\hline BrKMT3;4d & Bra007496 & 9 & & & & & & & & \\
\hline BrKMT6A;1 & Bra032169 & 4 & AtKMT6A;1 & SDG1/CLF & OsKMT6A;1 & SDG711 & BdKMT6A;1 & Bradi1g48340 & OtKMT6A & SDG3609 \\
\hline BrKMT6A;2 & Bra036300 & 9 & AtKMT6A;2 & SDG10/EZA1/SWN & OsKMT6A;2 & SDG718 & BdKMT6A;2 & Bradi1g64460 & & \\
\hline BrKMT6A;3a & Bra033334 & 10 & AtKMT6A;3 & SDG5/MEA & & & & & & \\
\hline BrKMT6A;3b & Bra032592 & 9 & & & & & & & & \\
\hline BrKMT6B;1a & Bra009403 & 10 & AtKMT6B;1 & SDG15/ATXR5 & OsKMT6B;1 & SDG720 & BdKMT6B;1 & Bradi2g61717 & & \\
\hline BrKMT6B;1b & Bra028618 & 2 & AtKMT6B;2 & SDG34/ATXR6 & OsKMT6B;2 & SDG730 & BdKMT6B;2 & Bradi3g02220 & & \\
\hline BrKMT6B;2a & Bra009752 & 6 & & & & & & & & \\
\hline BrKMT6B;2b & Bra029401 & 2 & & & & & & & & \\
\hline BrKMT7;1a & Bra037086 & 5 & AtKMT7;1 & SDG2/ATXR3 & OsKMT7;1 & SDG701 & BdKMT7;1 & Bradi3g15410 & OtKMT7;1a & SDG3606 \\
\hline BrKMT7;1b & Bra039562 & 1 & & & & & & & OtKMT7;1b & SDG3602 \\
\hline BrKMT7;1C & Bra020935 & 8 & & & & & & & & \\
\hline
\end{tabular}


Table 1 List of green lingeage SET-domain proteins analyzed in this study (Continued)

\begin{tabular}{|c|c|c|c|c|c|c|c|c|c|c|}
\hline BrS-ET;1 & Bra024688 & 9 & AtS-ET;1 & SDG35 & OsS-ET;1 & SDG739 & BdS-ET;1 & Bradi1g73790 & OtS-ET;1a & SDG3615 \\
\hline $\mathrm{BrS}-\mathrm{ET} ; 2$ & Bra028726 & 2 & AtS-ET;2 & SDG38 & OsS-ET;2 & SDG741 & BdS-ET;3 & Bradi5g22730 & OtS-ET;1b & SDG3613 \\
\hline$\overline{\mathrm{BrS}-\mathrm{ET} ; 3}$ & Bra031313 & 5 & AtS-ET;3 & SDG36 & OsS-ET;3 & SDG722 & BdS-ET;4a & Bradi3g17710 & OtS-ET;1C & SDG3611 \\
\hline BrS-ET;4a & Bra036713 & 9 & AtS-ET;4a & SDG39 & OsS-ET;4a & SDG740 & BdS-ET;4b & Bradi1g11010 & OtS-ET;3 & SDG3604 \\
\hline BrS-ET;4b & Bra002100 & 10 & AtS-ET;4b & SDG37 & OsS-ET;4b & SDG716 & & & & \\
\hline
\end{tabular}

*ud, undetermined

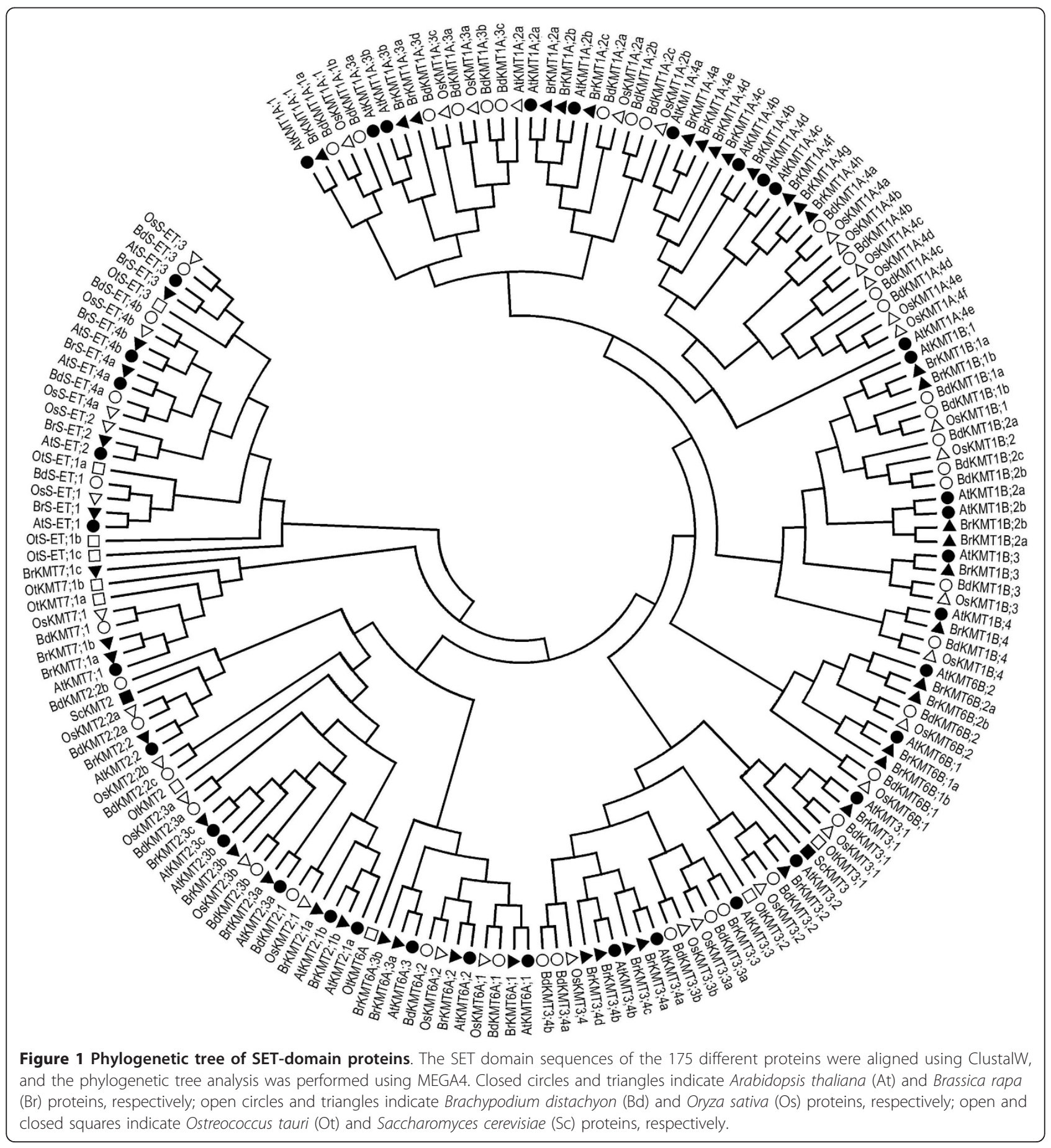




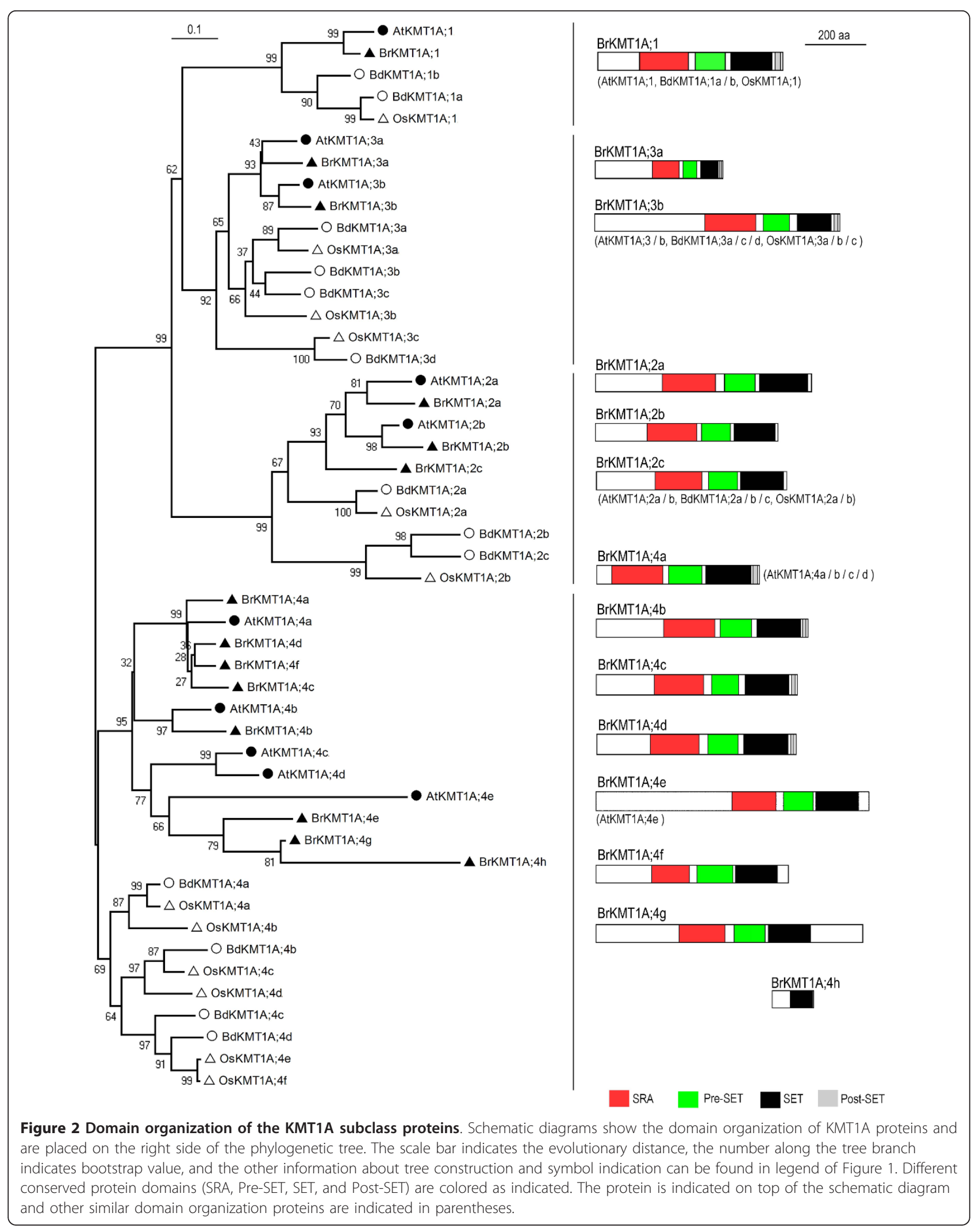


thaliana (http://brassicadb.org/brad/searchSynteny.php) revealed that BrKMT1A;4a, BrKMT1A;4c and BrKM $T 1 A ; 4 d$ but not BrKMT1A;4f are syntenic with AtKMT1A;4a/SDG32/SUVH1, and BrKMT1A;4 $h$ but not $\operatorname{BrKMT1A;4e}$ nor BrKMT1A;4 $g$ is syntenic with AtKMT1A;4e/SDG11/SUVH10. It thus appears that multiple duplication events occurred, in either a chromosome segment or single gene scale, resulting in more recent amplification of Group-4 genes in B. rapa after separation from $A$. thaliana during evolution. In agreement with previous studies in Arabidopsis, rice and maize [11,12], few introns are present in BrKMT1A genes (Additional File 1: Figure S1). Most BrKMT1A genes are represented by ESTs, but some do not have any ESTs in current databases (Additional File 2: Table S1). Our RT-PCR analysis revealed that indeed two genes that lack ESTs, BrKMT1A;2a and $B r K M T 1 A ; 2 c$, are very weakly expressed. Strong expression was detected for $B r K M T 1 A ; 4 a$, but relatively weak expres-

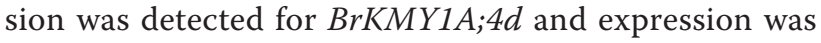
undetectable for $B r K M T 1 A ; 4 c$ (Additional File 3: Figure $\mathrm{S} 2$ ). Together, these data indicate that expression levels of different BrKMT1A genes varied considerably and thus these genes may regulate genome function to different degrees.

The plant KMT1A subclass proteins show high sequence similarity to the animal KMT1 proteins both within the SET domain and in the surrounding regions known as the Pre-SET and post-SET domains. Additionally, most of the plant proteins contain a specific domain named SRA (SET and RING associated). Similar to previously studied Arabidopsis proteins [12,20], most of the BrKMT1A proteins also contain SRA, Pre-SET, SET and post-SET domains (Figure 2). These domains are missing in some of the Group-4 proteins; for example, BrKMT1A.4e, BrKMT1A;4f and BrKMT1A;4 g lack a Post-SET domain, and BrKMT1A.4 h lacks SRA, PreSET and post-SET domains (Figure 2). Several functions have been reported for SRA domains, including binding with the $\mathrm{N}$-terminal tail of histone $\mathrm{H} 3$ and with DNA cytosine methylation [21]. The crystal structure of AtKMT1A;3a/SDG9/SUVH5 revealed that SRA recognizes the methylation status of $\mathrm{CG}$ and $\mathrm{CHH}$ sequences [22]. The Pre-SET domain contains 9 conserved cysteines. The Post-SET domain is a small cysteine-rich region often found at the $\mathrm{C}$-terminal side of SET domains. Both Pre-SET and Post-SET domains have been shown to affect histone methyltransferase activity of the SET domain [23,24].

Members of the plant KMT1A subclass, like animal KMT1 proteins, are likely to be responsible for H3K9 methylation, an epigenetic mark involved in heterochromatin formation and gene silencing. Consistent with this, analysis of AtKMT1A;1/SDG33/SUVH4/KYP, AtKMT1A;2a/SDG3/SUVH2, AtKMT1A;3a/SDG9/SUVH5 and
AtKMT1A;3b/SDG23/SUVH6 has revealed their important roles in H3K9 methylation, in heterochromatic gene silencing and in cross-talk between H3K9 and DNA methylation $[9,21,22,25-28]$. Work in rice also confirmed that several members of this subclass are involved in H3K9 methylation and in transposon silencing [29-31]. Some of the BrKMT1A genes might also have similar functions.

\section{The KMT1B subclass proteins}

Six B. rapa proteins belong to the KMT1B subclass, which can be further divided into 4 groups (Figure 3 ). Group-1 contains two B. rapa proteins (BrKMT1B;1a and BrKMT1B;1b) whose genes show synteny with AtKMT1B;1/SDG31/SUVR4. Moreover, sequence analysis showed that BrKMT1B;1b and AtKMT1B;1/SDG31/ SUVR4 have highly similar protein domain organization, indicating that $\operatorname{BrKMT1B;1b}$ is more conserved and $B r K M T 1 B ; 1 a$ diverged relatively late during evolution after the $B$. rapa/A. thaliana separation. Group-2 has two $B$. rapa, two $A$. thaliana proteins, one $O$. sativa protein, and three $B$. distachyon proteins. Both BrKMT1B;2a and $B r K M T 1 B ; 2 b$ show synteny with AtKMT1B;2b/ SDG18/SUVR2. Group-3 and 4 each have one representative member in each of the four examined higher plant species.

The KMT1B subclass differs from the KMT1A subclass in protein domain organization; specifically, these proteins lack the SRA domain (Figure 3). A recent study demonstrated that AtKMT1B;1/SDG31/SUVR4 possesses H3K9methyltransferase activities and its binding with ubiquitin converts H3K9me1 to H3K9me3 deposition on transposon chromatin [32]. Notably, the WIYLD domain, which binds ubiquitin, is conserved in BrKMT1B;1a, BrKMT1B;1b, BrKMT1B;2a and BrKMT1B;2b (Figure 3). It was reported that AtKMT1B;3/SDG6/SUVR5/AtCZS is involved in regulation of flowering time, possibly through deposition of H3K9 methylation at the flowering time repressor FLC [33]. The functions of other members of the KMT1B subclass remain uncharacterized so far.

\section{The KMT2 class proteins}

The KMT2 class includes six B. rapa and six A. thaliana proteins in 3 groups (Figure 4). This class features highly conserved SET and Post-SET domains with the yeast H3K4-methyltransferase ScKMT2/Set1. Nevertheless, some plant proteins have acquired specific domains during evolution, namely PWWP, PHD, FYR and/or GYF. The PWWP domain is also found in eukaryotic proteins involved in DNA methylation, DNA repair, and regulation of transcription [34], and regulates cell growth and differentiation by mediating protein-protein interactions [35]. The PHD domain is found in a number of chromatinassociated proteins and is thought to be involved in protein-protein interactions important for the assembly of 


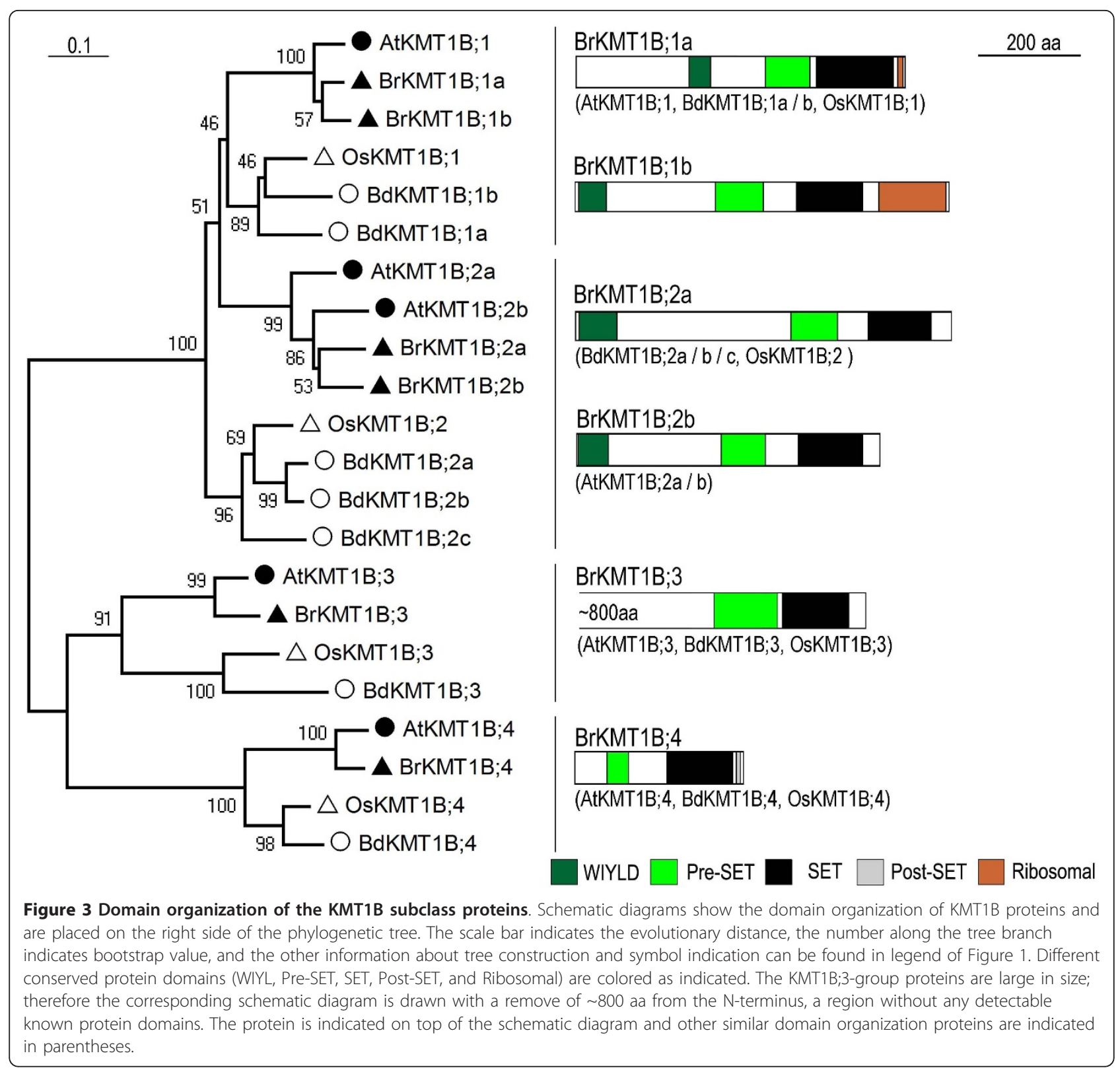

multiprotein complexes [36]. The PWWP domain of the animal BRPF1 protein binds H3K36me3 [35], and the PHD domain is also an important module in proteins that read histone modifications [37]. The FYR domain is composed of FYR-C and FYR-N terminal portions, which are often located close to each other but can also be separated [38]. The GYF domain is proposed to be involved in recognition of proline-rich sequences in protein-protein interactions [39].

KMT2 Group-1 members contain one PWWP, one FYR and two PHD domains. Only one member belonging to Group-1 is found in O. sativa or B. distachyon, but two members are found in B. rapa and A. thaliana. Our examination revealed that $B r K M T 2 ; 1 a$ has synteny with
AtKMT2;1a/SDG27/ATX1, and BrKMT2;1b with AtKM $T 2 ; 1 b / S D G 30 / A T X 2$, suggesting that they are derived from two different ancestral copies before the B. rapa/A. thaliana separation. Consistent with this, the atx 1 mutant plants exhibit strong and pleiotropic defects [40], but the atx2 mutant plants have a normal phenotype [41]. The atx 2 mutation can enhance atx 1 in reduction of expression of the flowering repressor gene $F L C$ through reduced levels of H3K4me3 at the FLC locus [42].

The PWWP and FYR domains are absent from the Group-2 members and the PHD domain is found only in some monocot proteins (Figure 4). Only one Group-2 representative member is found in the dicot species B. rapa or $A$. thaliana, but the monocot $O$. sativa has two 


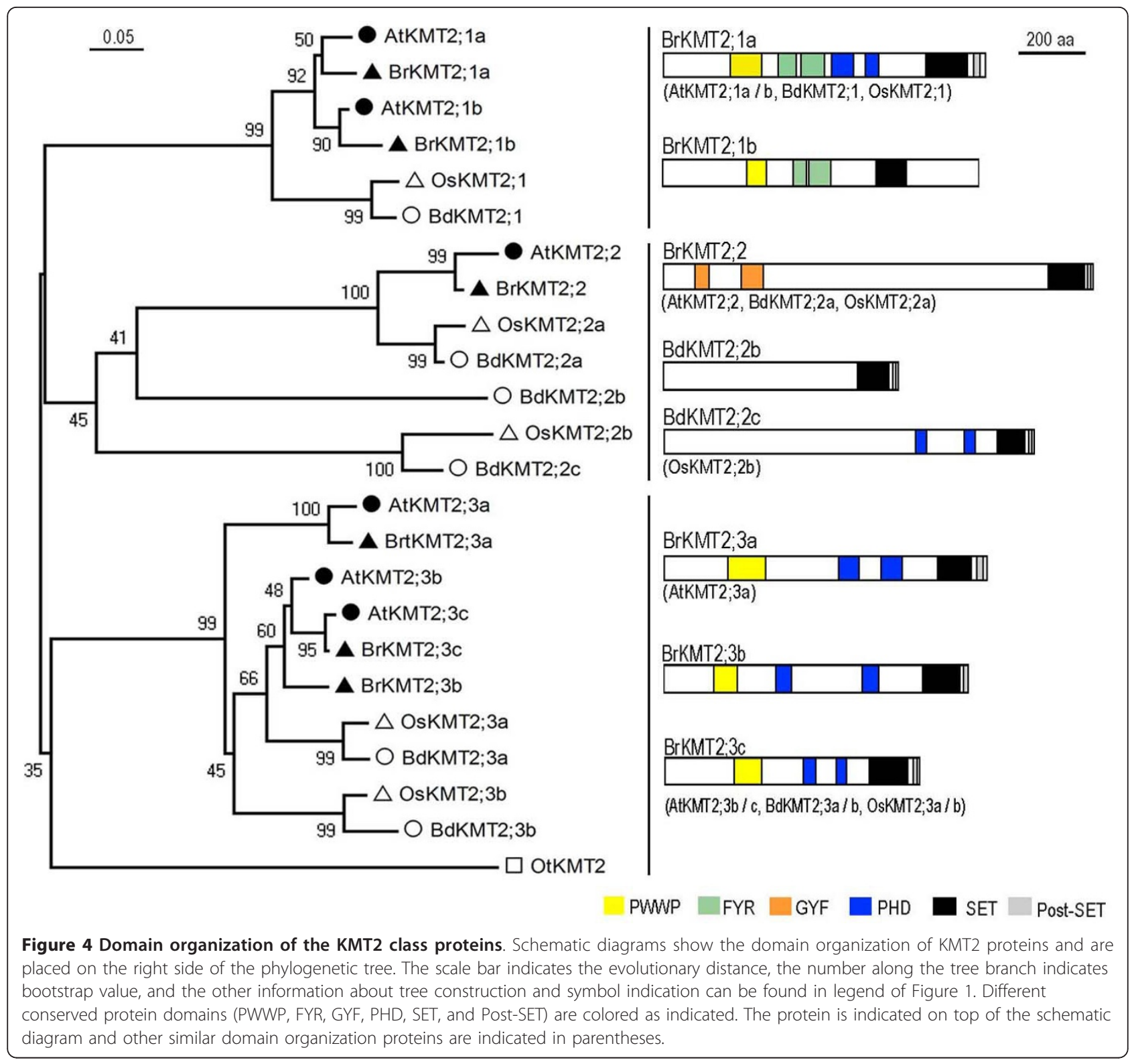

members and $B$. distachyon has three members. The $B$. rapa and $A$. thaliana proteins, as well as one member each from $O$. sativa and $B$. distachyon, contain a GYF domain in the $\mathrm{N}$-terminal part of the protein (Figure 4). The fact that this domain is conserved in KMT2;2 proteins from all four higher plant species suggests that the acquisition of the GYF domain occurred before the monocot/ dicot separation and may have a conserved function in higher plants. Genetic analysis demonstrated that AtKMT2;2/SDG25/ATXR7 is necessary in preventing early flowering [43,44]. The recombinant AtKMT2;2/SDG25/ ATXR7 protein was shown to methylate histone $\mathrm{H} 3$ in vitro and the depletion of AtKMT2;2/SDG25/ATXR7 in planta slightly reduced $\mathrm{H} 3 \mathrm{~K} 4$ and $\mathrm{H} 3 \mathrm{~K} 36$ methylation at
FLC chromatin [43,44]. OsKMT2;2b/SDG732 and BdKMT2;2c contain two PHD domains, but BdKMT2;2b, like the yeast protein ScKMT2/Set1, does not contain a recognizable PHD domain. Future study of these monocot proteins will likely provide a deeper understanding of the domain evolution of KMT2 proteins.

The Group-3 KMT2 proteins have a domain organization more similar to Group-1 except that they lack the FYR domain (Figure 4). B. rapa and A. thaliana both have three Group-3 members, but each of the other two higher plant species has only two Group-3 members. Synteny was observed for BrKMT2;3c with AtKMT2;3c/ SDG29/ATX5 but not with AtKMT2;3b/SDG16/ATX4, suggesting that $A t K M T 2 ; 3 b / S D G 16 / A T X 4$ was derived 
from a relatively recent duplication event. This is in agreement with a previous study revealing that $A t K$ $M T 2 ; 3 b / S D G 16 / A T X 4$ and AtKMT2;3c/SDG29/ATX5 are collinearly duplicated with AtKMT2;1a/SDG27/ATX1 and $A t K M T 2 ; 1 b / S D G 30 / A T X 2$ [12]. To date, none of the Group-3 proteins has been functionally characterized.

\section{The KMT3 class proteins}

The KMT3 class contains 5 members in A. thaliana but 7 members in B. rapa, and these can be further divided into four groups (Figure 5). The other groups contain a single member per plant species, but Group-4 contains 2 members in $A$. thaliana and 4 members in B. rapa. Our examination indicates that $B r K M T 3 ; 4 a$ and $B r K$ $M T 3 ; 4 c$ are syntenic with AtKMT3;4a/SDG7/ASHH3, and BrKMT3;4b and BrKMT3;4d with AtKMT3;4b/ SDG24/ASHH4. The ESTs found in the current databases match all four BrKMT3;4 genes (Additional File 2: Table S1), and thus do not allow us to distinguish expression of each gene. Our RT-PCR analysis indicated that BrKMT3;4a and BrKMT3;4c are expressed at higher levels and more broadly in different examined organs/

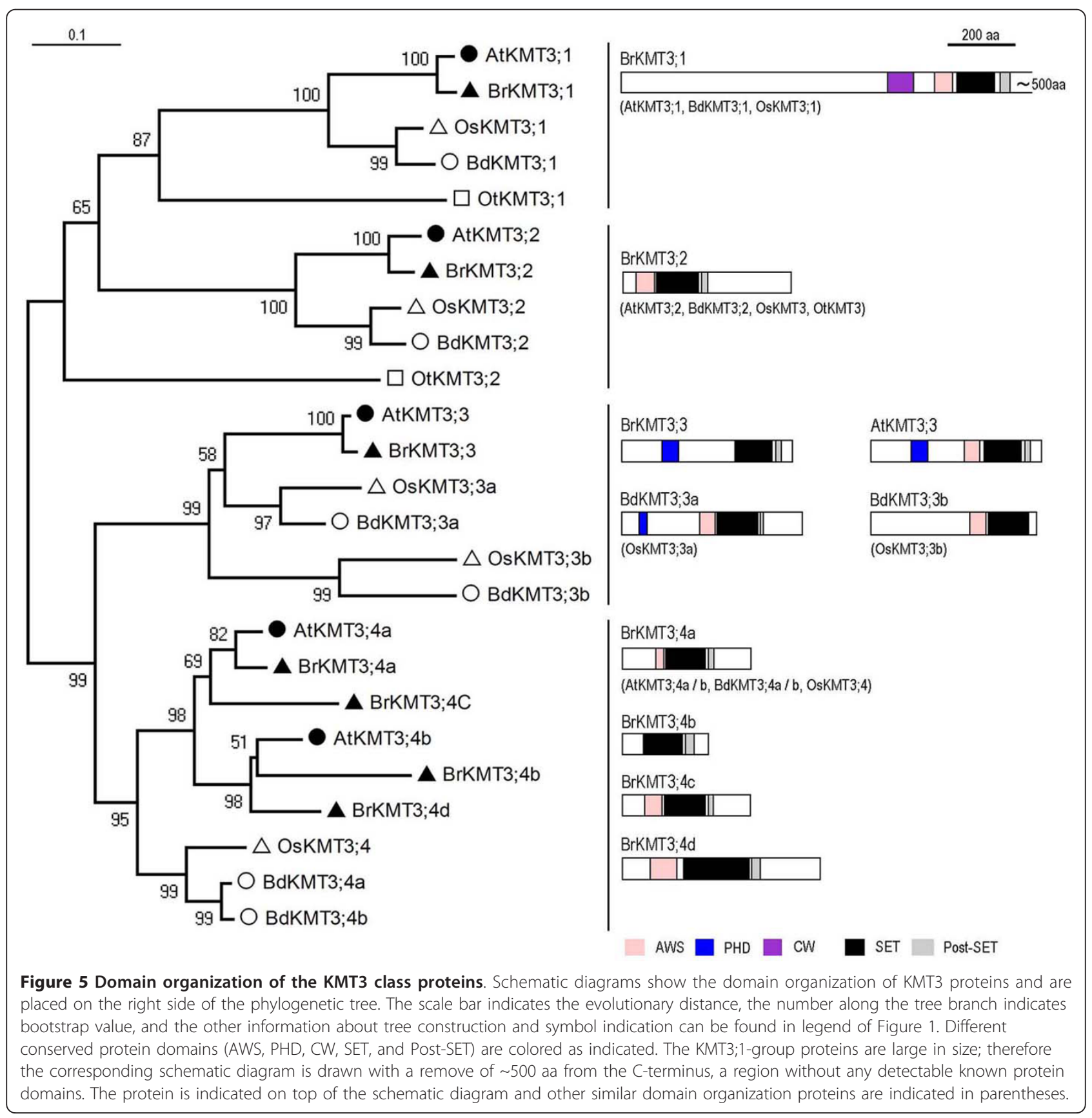


tissues, whereas only weak expression was detected for BrKMT3;4b and BrKMT3;4d in some organs/tissues (Additional File 3: Figure S2).

The KMT3 class plant proteins share high sequence similarity and share the AWS (a subdomain of Pre-SET, [45]), SET and Post-SET domain organization with the yeast H3K36-methyltransferase ScKMT3/Set2 (Figure 5). The Group-1 proteins have a long sequence and contain an additional CW domain specific to this group. The $\mathrm{CW}$ domain of AtKMT3;1/SDG8/ASHH2/EFS/CCR1 was recently shown to bind $\mathrm{H} 3 \mathrm{~K} 4 \mathrm{me} 1 / \mathrm{me} 2$ [46], suggesting a novel link between H3K4 and H3K36 methylation in plants. AtKMT3;1/SDG8/ASHH2/EFS/CCR1 is the major H3K36-methyltransferase specifically required for H3K36me 2 and H3K36me3 deposition, and activates expression of hundreds of genes including $F L C$ and $M A F \mathrm{~s}$ [47]. Depletion of AtKMT3;1/SDG8/ASHH2/EFS/CCR1 causes pleiotropic phenotypes, including early flowering, reduced organ size, increased shoot branching, perturbed fertility and carotenoid composition, and impaired plant defenses against pathogens [47-54]. The other group of KMT3 plant proteins have a shorter sequence and do not contain the $\mathrm{CW}$ domain; interestingly the depletion of AtKMT3;2/SDG26/ASHH1 resulted in a late-flowering phenotype associated with elevated levels of $F L C$ expression [47]. The Group-3 KMT3 proteins, with the exception of BdKMT3;3b and OsKMT3;3b/SDG707, contain a PHD domain; and AtKMT3;3/SDG4/ASHR3 was reported to be involved in pollen and stamen development possibly through mediating H3K4me2 and H3K36me3 deposition $[55,56]$. The functions of the Group-4 proteins remain unexamined so far. Examination of this group in B. rapa could be a challenge because of gene multiplication and more diverged sequences (Figure 5).

\section{The KMT6A subclass proteins}

The KMT6A subclass includes 4 members in B. rapa and 3 well-characterized members in A. thaliana, AtKMT6A;1/SDG1/CLF, AtKMT6A;2/SDG10/EZA1/ SWN and AtKMT6A;3/SDG5/MEA, which represent three distinct groups (Figure 6a). AtKMT6A;1/SDG1/ CLF and AtKMT6A;2/SDG10/EZA1/SWN are broadly expressed and partially redundant in regulation of vegetative and reproductive development, whereas $A t K$ $M T 6 A ; 3 / S D G 5 / M E A$ appears to function specifically in gametophyte and seed development $[7,8,57]$. The three Arabidopsis proteins can act as a key component of the evolutionarily conserved Polycomb Repressive Complex 2 (PRC2), which trimethylates H3K27 involved in transcriptional repression [57]. AtKMT6A;1/SDG1/CLF and AtKMT6A;2/SDG10/EZA1/SWN represent Group-1 and Group-2, respectively, and each has an orthologue in different higher plant species. AtKMT6A;3/SDG5/ MEA, which belongs to Group-3, has no orthologue in monocots but has two orthologues in $B$. rapa. Both $B r K M T 6 A ; 3 a$ and $B r K M T 6 A ; 3 b$ are syntenic with AtKMT6A;3/SDG5/MEA, and BrKMT6A;3a has a more similar protein domain organization to AtKMT6A;3/ SDG5/MEA than does BrKMT6A;3b, suggesting that $B r K M T 6 A ; 3 b$ may have diverged after the $A$. thaliana/B. rapa separation during evolution.

The SANT (SWI3, ADA2, N-CoR, and TFIIIB DNAbinding) domain is found in most of the plant KMT6A subclass proteins. This domain is also found in a number of other chromatin remodeling proteins with multiple activities such as DNA-binding, histone tail binding, and protein-protein interactions [58]. Nevertheless, the precise role of the SANT domain in KMT6A proteins is currently unknown. Notably, BrKMT6A;3b does not contain a SANT domain. As expected from restricted AtKMT6A;3/ $S D G 5 / M E A$ expression in only a small number of cells during reproduction, expression of both $B r K M T 6 A ; 3 a$ and $B r K M T 6 A ; 3 b$ is barely detectable in the examined tissues (Additional File 3: Figure S2). It will be interesting to investigate $B r K M T 6 A ; 3 a$ and $B r K M T 6 A ; 3 b$ expression during reproduction and to examine whether both genes are functionally important.

\section{The KMT6B subclass proteins}

The KMT6B subclass includes two members each in $A$. thaliana, $B$. distachyon and $O$. sativa, and four members in $B$. rapa, which together can be divided into two distinct groups (Figure $6 \mathrm{~b}$ ). The two members from $A$. thaliana, AtKMT6B;1/SDG15/ATXR5 and AtKMT6B;2/SDG34/ ATXR6, were classified as trithorax-related in the first genome analysis of Arabidopsis SET-domain proteins [11]. However, our study as well as two previous studies that included a more complete set of plant SET-domain proteins clearly show that AtKMT6B;1/SDG15/ATXR5 and AtKMT6B;2/SDG34/ATXR6 belong to the KMT6B subclass (Figure 1) $[12,20]$. Consistent with this, functional analysis revealed that AtKMT6B;1/SDG15/ATXR5 and AtKMT6B;2/SDG34/ATXR6 are involved in monomethylation of H3K27 [59]. They appear to act redundantly, because depletion of H3K27 monomethylation is only detectable in the atxr5 atxr6 double mutant [59]. KMT6A-mediated H3K27me3 is mainly present in euchromatic regions and is important for gene silencing [58], but KMT6B-mediated H3K27me1 is found in heterochromatic chromocenters and is important for heterochromatin condensation and replication in Arabidopsis [60].

Distinct from KMT6A proteins containing a SANT domain, many plant KMT6B subclass proteins contain a PHD domain (Figure 6). The PHD domain of both AtKMT6B;1/SDG15/ATXR5 and AtKMT6B;2/SDG34/ ATXR6 strongly bind unmethylated $\mathrm{H} 3$ tail peptides (amino acids 1-21), and this binding is negatively affected 


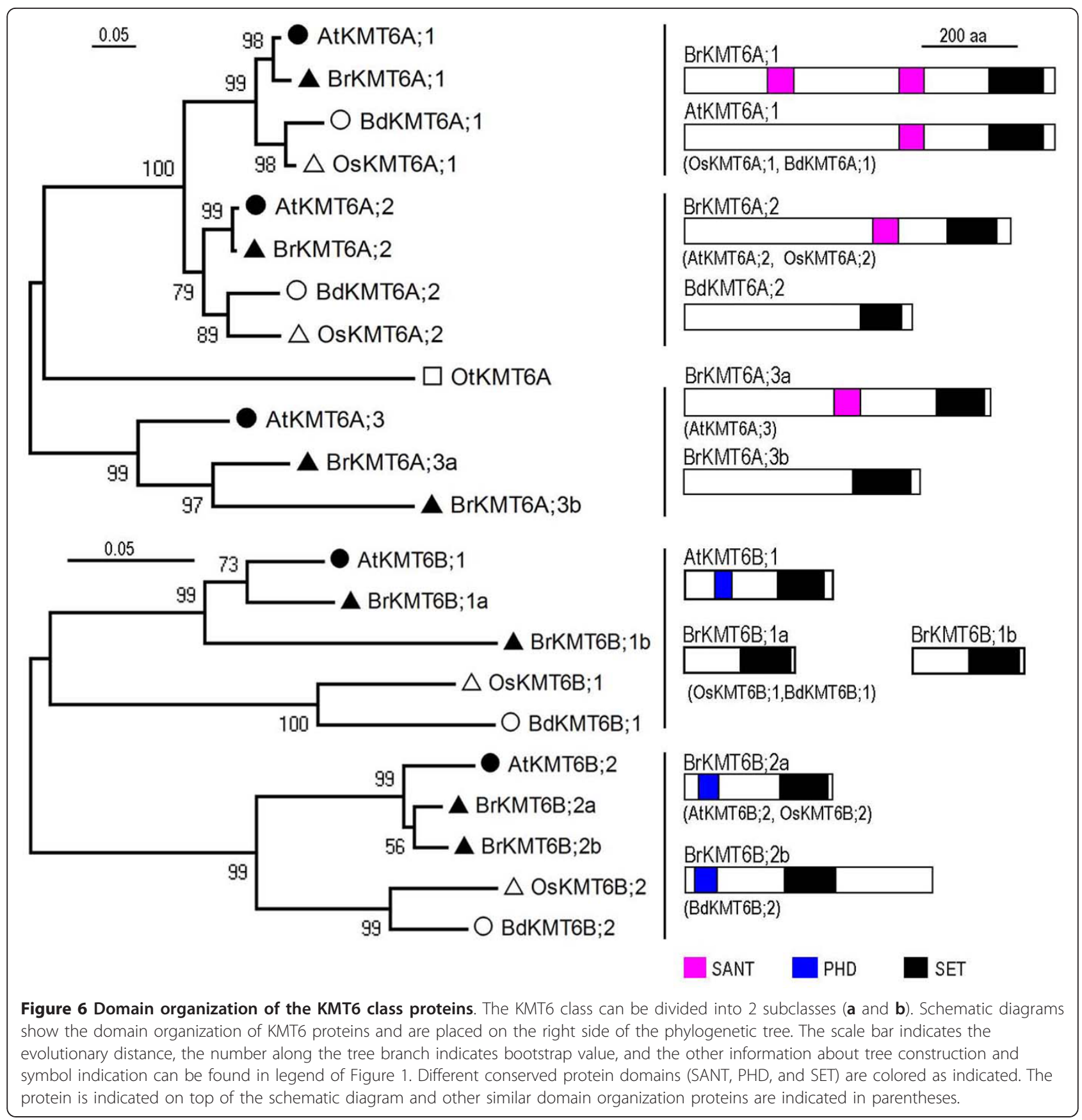

by methylation on H3K4 [60]. This binding preference may help to assure that these KMT6B proteins are not targeted to euchromatin and active genes enriched in H3K4 methylation. Remarkably, both Group-1 and Group-2 members are duplicated in $B$. rapa. Both $B r K M T 6 B ; 1 a$ and $B r K M T 6 B ; 1 b$ are syntenic with AtKMT6B;1/SDG15/ $A T X R 5$, and both BrKMT6B;2a and BrKMT6B;2b with AtKMT6B;2/SDG34/ATXR6. Expression of BrKMT6B;1a, BrKMT6B;2a and BrKMT6B;2b was detected in different tissues, but we failed to detect $B r K M T 6 B ; 1 b$ expression
(Additional File 3: Figure $\mathrm{S} 2$ ). It is reasonable to speculate that BrKMT6B;1a, BrKMT6B;2a and BrKMT6B;2b might have redundant functions.

\section{The KMT7 class proteins}

The KMT7 class contains a single member each in $A$. thaliana, $O$. sativa and $B$. distachyon, but three members in $B$. rapa (Figure 7). Although the Arabidopsis protein AtKMT7;1/SDG2/ATXR3 was considered to be related to members of the KMT2 class in some previous studies 


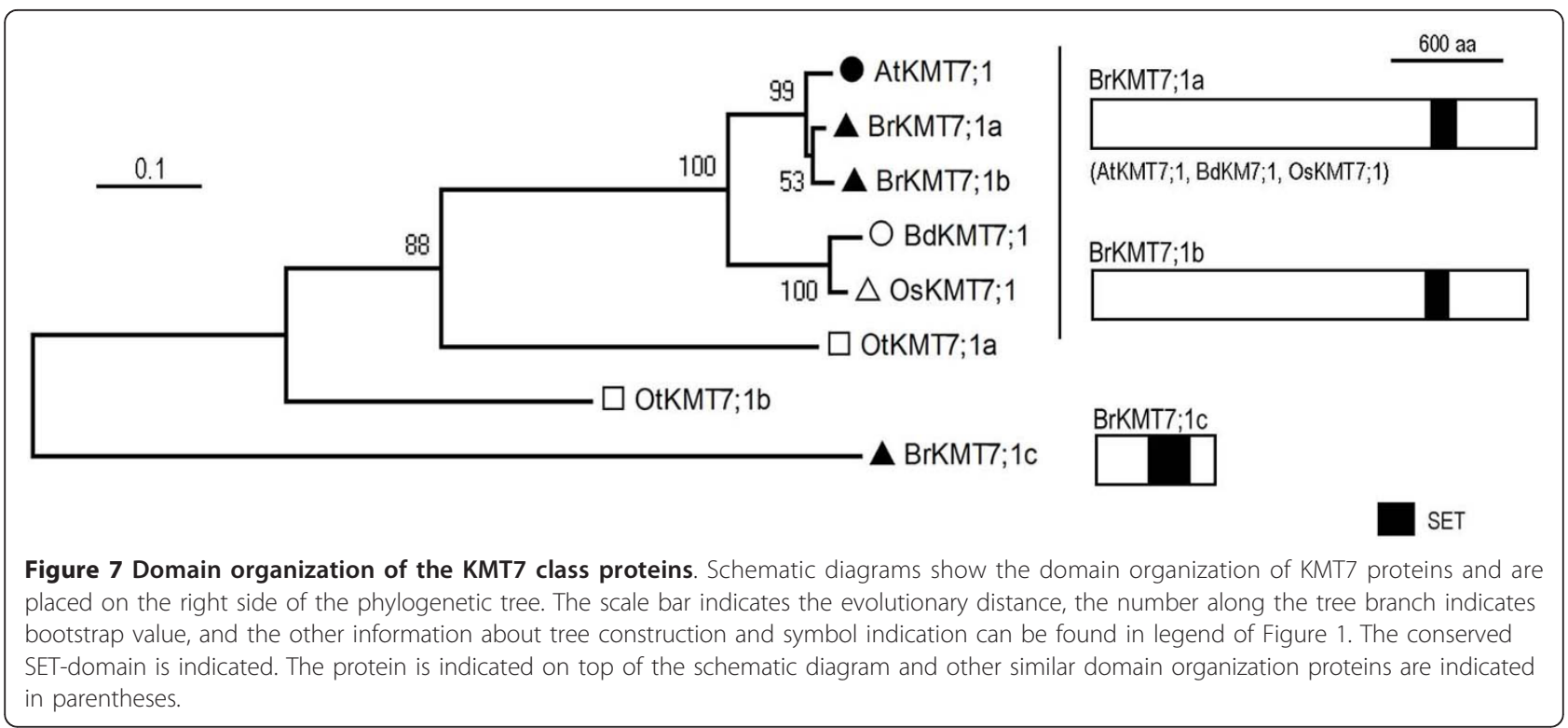

$[11,20]$, it was located outside of any classes in the phylogenetic tree analysis by Springer and colleagues [12], and our analysis here revealed that it is grouped together with some other green lineage proteins, forming the plant KMT7 class (Figure 1). Unlike the classes described above, the plant and animal KMT7 classes do not cluster although they are predicted to have similar functions in H3K4 methylation. Representatives of the animal KMT7 class are only found in mammals and include the human SET7/9, which monomethylates H3K4 and also methylates a number of nonhistone proteins [17]. The plant KMT7 proteins did not show the highest sequence similarities with the human SET7/9, and depletion of AtKMT7;1/SDG2/ATXR3 resulted in a global reduction of H3K4me3 and caused pleiotropic defects in both sporophyte and gametophyte development $[61,62]$. Both BrKMT7;1a and BrKMT7;1b but not BrKMT7;1c have synteny with $A t K M T 7 ; 1 / S D G 2 / A T X R 3$, and phylogenetic analysis showed that BrKMT7;1a is more closely related to AtKMT7;1/SDG2/ATXR3. RT-PCR analysis revealed that $\operatorname{BrKMT7;1b}$ is expressed at a higher level than $B r K M T 7 ; 1 a$ (Additional File 3: Figure S2). In view of the important function of $A t K M T 7 ; 1 a / S D G 2 / A T X R 3$, it will be interesting to investigate roles of $B r K M T 7 ; 1 a$ and $B r K M T 7 ; 1 b$ in histone methylation and plant development in B. rapa.

\section{Discussion}

Over last 10 years, a number of SET-domain genes in Arabidopsis and in rice have been characterized and shown to exert crucial chromatin-based functions via histone methylation during plant growth and development $[3,15]$. However, the nomenclature of plant SET-domain proteins remains complex, and multiple synonyms exist for many Arabidopsis proteins (Table 1), which could cause considerable confusion in this important field. Nomenclature based on sequence similarity has several advantages, informing the prediction of KMT enzyme substrate specificity for its histone lysine residue and providing a global view of KMT types in an organism once its whole genome sequence becomes available. However, the SDG nomenclature failed to provide information concerning enzyme substrate specificity and the number following "SDG" could be long and difficult to remember $[12,63]$, e.g., the first SDG from B. rapa (ID = 197) would have been named SDG19701. While the nomenclature by Baumbusch and colleagues provided information about homology to animal proteins, an incomplete list of Arabidopsis SET-domain proteins and the limitation (at that time) of having only one plant species with a genome-wide analysis restricted the precision and correctness of phylogenetic grouping in this study [11]. In addition, animal KMT nomenclature had also been noncoherent; a rational nomenclature was proposed only recently [17]. Therefore, the nomenclature we propose here is in line with the latest advances in the field.

In accordance with the guidelines of the Commission on Plant Gene Nomenclature [64], the nomenclature of plant KMTs is defined by species initials (e.g. Br for Brasica rapa) before KMT, which is followed by the class number (Figure 8). The class number is based on the yeast and animal systems indicating the enzyme substrate specificity, i.e. KMT1 for H3K9, KMT2 for H3K4, KMT3 for H3K36, KMT6 for H3K27, and KMT7 also for H3K4 [17]. Multiple subclasses are indicated by upper-case letters (e.g. KMT1A and KMT1B), and distinct groups 


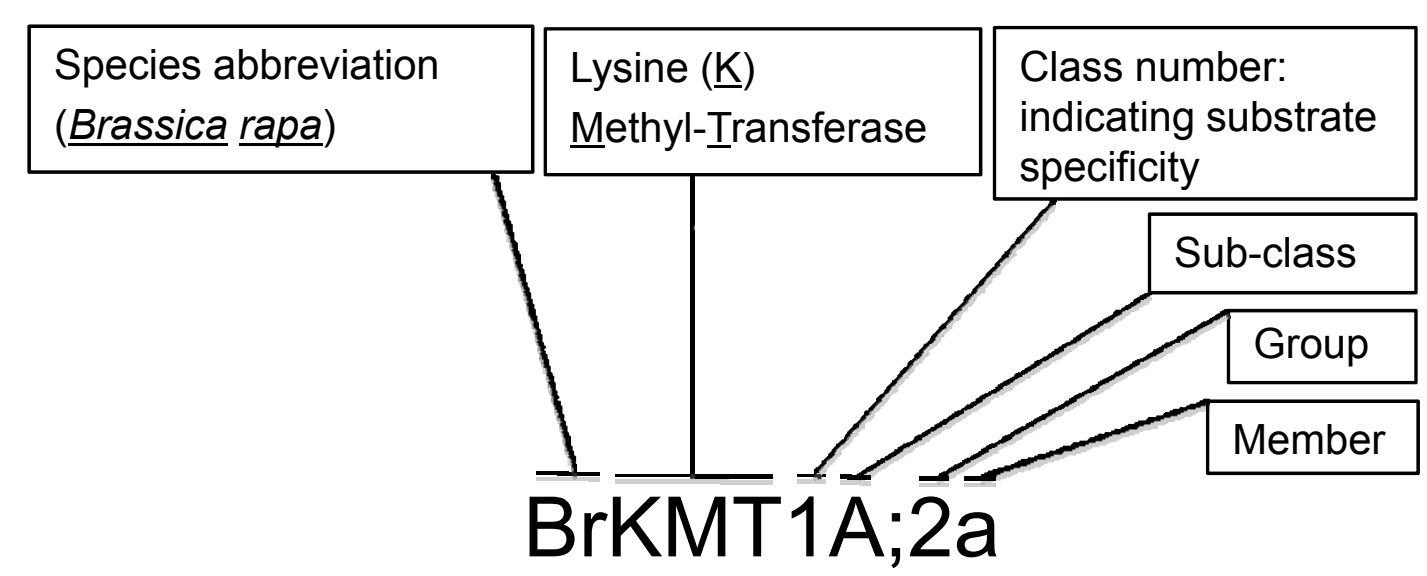

Figure 8 Nomenclature for plant KMTs. The BrKMT1A;2a protein serves as an example to show assignment of various layers of information within the nomenclature of a plant KMT. Refer to text for class, subclass, group and member definition.

within the class/subclass are indicated by an arabic numeral suffix (e.g. KMT1A;1). Members within the group are indicated by lower-case letters (e.g. KMT1A;1a and KMT1A;1b). Subgroups are not currently defined but may be designated in the future as functional analysis and an increasing number of sequenced genomes demonstrate sequence conservation between species or distinct functions for several members of a defined KMT group. The use of a given subgroup suffix should indicate highly similar sequences or equivalent functional roles between several species. The new nomenclature may be difficult to adopt in Arabidopsis because the original names for a number of SET-domain proteins are familiar to researchers, but a coherent and rational nomenclature for different species is important and useful because of the enormous interest in KMTs. The guidelines proposed here will be particularly useful for nomenclature of newly identified SET-domain proteins, which are being discovered at an exponentially increasing rate as genome sequences become available for additional plant species.

We identified 49 SET-domain proteins from the recently completed whole genome sequence of $B$. rapa. Among them, 5 proteins belong to the S-ET class likely involved in nonhistone protein methylation, 20 proteins belong to the KMT1 class potentially involved in H3K9 methylation, 6 proteins belong to the KMT2 class potentially involved in $\mathrm{H} 3 \mathrm{~K} 4$ methylation, 7 proteins belong to the KMT3 class potentially involved in H3K36 methylation, 8 proteins belong to the KMT6 class potentially involved in H3K27 methylation, and 3 belong to the KMT7 class also potentially involved in H3K4 methylation. This in silico survey is useful for future functional analysis of this important family of epigenetic regulators in Brassica. H4K20 methylation was detected in Arabidopsis using antibodies $[28,65]$, but the catalyzing enzyme(s) involved is(are) not yet known and the current phylogenetic analysis did not allow prediction of a specific KMT class involved in H4K20 methylation. It is possible that some members of the aforementioned KMT classes catalyze H4K20 methylation. The total number of KMTs in B. rapa (49) is slightly higher than that identified in A. thaliana (37), O. sativa (36) and B. distachyon (41). Nevertheless, we could not exclude the possibility that a few more KMTs may be missing from the currently available genome sequence of B. rapa.

Gene duplication is one of the primary driving forces in the evolution of genomes and genetic systems, and is considered to be a major mechanism for the establishment of new gene functions and the generation of evolutionary novelty $[66,67]$. Contrary to what would be expected from the chromosome number duplication in B. rapa compared to $A$. thaliana, the number of KMT genes in B. rapa (49) is much less than double the number of $A$. thaliana KMTs (74). Many duplicated genes show synteny with their $A$. thaliana homologues, suggesting that they are derived from chromosome/genome segment duplications. Three alternative outcomes can occur in the evolution of duplicated genes: (i) one copy may simply become silenced by degenerative mutations (nonfunctionalization); (ii) one copy may acquire a novel, beneficial function and become preserved by natural selection (neofunctionalization); (iii) both copies may become partially compromised by mutation so that their total capacity adds up to the capacity of the single-copy ancestral gene (subfunctionalization) [66]. These different outcomes likely apply to different duplicated KMT genes, judging from their expression patterns (Additional File 3: Figure S2). Expression of BrKMT1A;4c and $B r K M T 6 B ; 1 b$ was undetectable, suggesting that they might have been nonfunctionalized. The duplicated pairs $B r K M T 1 B ; 2 a$ and BrKMT1B;2b, BrKMT3;4b and BrK MT3;4d, or BrKMT7;1 $a$ and BrKMT7;1b are differentially expressed in plant organs, suggesting that they might have 
acquired distinct tissue-specific functions. Finally, expression of some duplicated genes, e.g. BrKMT1A;2a and BrKMT1A;2c, BrKMT1B;1a and BrKMT1B;1b, or BrK $M T 6 A ; 3 a$ and $B r K M T 6 A ; 3 b$ showed similar patterns, suggesting that they might be subfunctionalized and/or have redundant functions.

Among the groups showing gene duplications in $B$. rapa, it is worth to note that in Arabidopsis, AtKMT6A;3/ $S D G 5 / M E A$ is critical for parental gene imprinting and seed development [8,68], AtKMT6B;1/SDG15/ATX5 and AtKMT6B;2/SDG34/ATX6 are important for heterochromatin condensation and replication in Arabidopsis [59,60], and AtKMT7;1/SDG2/ATXR3 is essential for both sporophyte and gametophyte development $[61,62]$. It will be of great interest to investigate these groups of genes for their regulation and function in chromatin organization, plant growth and development in B. rapa.

\section{Conclusions}

Our study shows that the plant SET-domain KMT proteins can be phylogenetically grouped into distinct classes and that the classes involved in histone methylation can be named in accordance with the nomenclature proposed for animal and yeast SET-domain KMTs. Such a coherent and rational nomenclature in different organisms will help avoid confusion caused by the existence of multiple names for the same protein or gene. The information provided on the $B$. rapa KMTs will also be beneficial for future research to unravel the mechanisms of epigenetic regulation in Brassica crops.

\section{Methods}

\section{SET-domain protein identification}

Sequences of SET-domain proteins from A. thaliana, $O$. sativa, $O$. tauri and $S$. cerevisiae were retrieved from the Chromatin Database with the key word SDG in species database respectively (ChromDB, http://www.chromdb. org). These sequences, primarily those from $A$. thaliana and $O$. sativa, were used as queries to search the $B$. distachyon genome (http://www.brachypodium.org) and the B. rapa genome (http://brassicadb.org/brad/index.php) by using the BLASTp and tBLASTn tools (http://blast.ncbi. nlm.nih.gov). The Expect threshold was set at 1.0 and other parameters were set at default values. We did not use a strict E-value threshold; rather we examined each of the resulting hits for the presence of the SET or S-ET domain to collect previously unidentified sequences. The synteny analysis was performed using the online viewer tool (http://brassicadb.org/brad/index.php). ESTs of the $B$. rapa SET-domain protein genes were retrieved from the Brassica Database (http://brassicadb.org/brad/index.php) and from NCBI (http://blast.ncbi.nlm.nih.gov), using an Expect threshold of 1, and a minimum sequence length of $50 \mathrm{bp}$.

\section{Protein domain organization analysis}

The protein sequences were analyzed for domain organization using NCBI-CD searches (http://ncbi.nlm.nih. gov/Structure/cdd/wrpsb.cgi). The low-complexity filter was turned off, and the Expect value was set at 1.0 to detect short domains or regions of less conservation in this analysis. Domains were also verified and named according to the SMART database (http://smart.emblheidelberg.de/).

\section{Phylogenetic analysis}

Multiple sequence alignments of SET-domain sequences were performed using the ClustalW program [69]. The resulting file was subjected to phylogenic analysis using the MEGA4.0 program [70]. The trees were constructed with the following settings: Tree Inference as NeighborJoining; Include Sites as pairwise deletion option for total sequences analysis and complete deletion option for each class analysis; Substitution Model: Poisson correction; and Bootstrap test of 1,000 replicates for internal branch reliability.

\section{RT-PCR Analysis}

B. rapa plants were grown at $18-22^{\circ} \mathrm{C}$ under a $12 \mathrm{~h}$ light $(10,000 \mathrm{Lx}) / 12 \mathrm{~h}$ dark photoperiod. Leaves were collected from 2-, 4-, 6-, 8- or 10-week-old plants; roots and stems were collected from 6-week-old plants; flower buds were collected from 10-week-old plants. Total RNA was extracted using Trizol reagent (Invitrogen, USA) from about $100 \mathrm{mg}$ of collected plant tissue. The RNA preparation was then treated with DNaseI (Promega, USA) for $30 \mathrm{~min}$ at $37^{\circ} \mathrm{C}$, followed by enzyme inactivation by incubation at $65^{\circ} \mathrm{C}$ for $5 \mathrm{~min}$. First strand cDNA was made using an RT-PCR Kit (RevertAid ${ }^{\mathrm{TM}}$ First Strand cDNA Synthesis Kit, Fermentas, CA). The RT-solution with first strand cDNA was stored at $-80^{\circ} \mathrm{C}$. Primers used for the $\mathrm{RT}$-PCR reactions are listed in Additional File 4: Table S2. Conditions for the PCR reactions were as follows: $94^{\circ} \mathrm{C}$ for $3 \mathrm{~min}$; then 30 cycles of $94^{\circ} \mathrm{C}$ for $30 \mathrm{~s}, 50-63^{\circ} \mathrm{C}$ for $30 \mathrm{~s}$, and $72^{\circ} \mathrm{C}$ for $1 \mathrm{~min}$; and finally $72^{\circ} \mathrm{C}$ for $8 \mathrm{~min}$. PCR products were separated in a $1.5 \%(\mathrm{w} / \mathrm{v})$ agarose Tris-borate/EDTA buffer gel and visualized by ethidium bromide staining.

\section{Additional material}

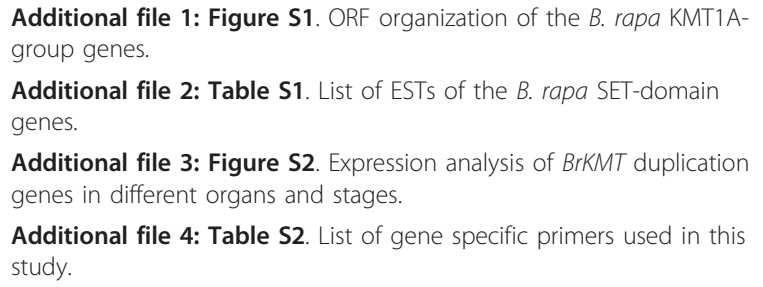




\section{Acknowledgements}

We thank Dr Xiaowu Wang, Jian Wu and Fen Chen, from the Institute of Vegetables and Flowers of the Chinese Academy of Agricultural Sciences, for providing sequences and helping in synteny analysis. This work was supported in part by National Basic Research Program of China (973 Program, 2012CB910500) and National Natural Science Foundation of China (NSFC31071129 and NSFC31071455).

\section{Author details}

${ }^{1}$ Hunan Provincial Key Laboratory of Crop Germplasm Innovation and Utilization, Hunan Agricultural University, Changsha 410128, China. ${ }^{2}$ College of Bioscience and Biotechnology, Hunan Agricultural University, Changsha 410128, China. ${ }^{3}$ Institut de Biologie Moléculaire des Plantes du CNRS, Université de Strasbourg, 12 rue du Général Zimmer, 67084 Strasbourg Cedex, France.

\section{Authors' contributions}

$\mathrm{YH}$ conducted most of the experiments and drafted the manuscript; $\mathrm{CL}$ contributed to the RT-PCR experiment and participated in the drafting of the manuscript; WHS and YR conceived and directed the study, and wrote the final version of the manuscript. All authors read and approved the final manuscript.

Received: 4 November 2011 Accepted: 14 December 2011

Published: 14 December 2011

\section{References}

1. Strahl $B D$, Allis CD: The language of covalent histone modifications. Nature 2000, 403:41-45.

2. Martin C, Zhang Y: The diverse functions of histone lysine methylation. Nat Rev Mol Cell Biol 2005, 6:838-849.

3. Liu C, Lu F, Cui X, Cao X: Histone methylation in higher plants. Annu Rev Plant Biol 2010, 61:395-420

4. Tschiersch B, Hofmann A, Krauss V, Dorn R, Korge G, Reuter G: The protein encoded by the Drosophila position-effect variegation suppressor gene Su(var)3-9 combines domains of antagonistic regulators of homeotic gene complexes. EMBO J 1994, 13:3822-3831.

5. Qian C, Zhou MM: SET domain protein lysine methyltransferases: structure, specificity and catalysis. Cell Mol Life Sci 2006, 63:2755-2763.

6. Alvarez-Venegas R, Sadder M, Tikhonov A, Avramova Z: Origin of the bacterial SET domain genes: vertical or horizontal? Mol Biol Evol 2007, 24:482-497.

7. Goodrich J, Puangsomlee P, Martin M, Long D, Meyerowitz EM, Coupland G: A Polycomb-group gene regulates homeotic gene expression in Arabidopsis. Nature 1997, 386:44-51.

8. Grossniklaus U, Vielle-Calzada JP, Hoeppner MA, Gagliano WB: Maternal control of embryogenesis by MEDEA, a polycomb group gene in Arabidopsis. Science 1998, 280:446-450.

9. Jackson JP, Lindroth AM, Cao X, Jacobsen SE: Control of CpNpG DNA methylation by the KRYPTONITE histone H3 methyltransferase. Nature 2002, 416:556-560

10. Shen WH: NtSET1, a member of a newly identified subgroup of plant SET-domain-containing proteins, is chromatin-associated and its ectopic overexpression inhibits tobacco plant growth. Plant J 2001, 28:371-383.

11. Baumbusch LO, Thorstensen T, Krauss V, Fischer A, Naumann K, Assalkhou R, Schulz I, Reuter G, Aalen RB: The Arabidopsis thaliana genome contains at least 29 active genes encoding SET domain proteins that can be assigned to four evolutionarily conserved classes. Nucleic Acids Res 2001, 29:4319-4333.

12. Springer NM, Napoli CA, Selinger DA, Pandey R, Cone KC, Chandler VL, Kaeppler HF, Kaeppler SM: Comparative analysis of SET domain proteins in maize and Arabidopsis reveals multiple duplications preceding the divergence of monocots and dicots. Plant Physiol 2003, 132:907-925.

13. Pien $\mathrm{S}$, Grossniklaus U: Polycomb group and trithorax group proteins in Arabidopsis. Biochim Biophys Acta 2007, 1769:375-382.

14. Shen WH, Xu L: Chromatin remodeling in stem cell maintenance in Arabidopsis thaliana. Mol Plant 2009, 2:600-609.

15. Berr A, Shafiq S, Shen WH: Histone modifications in transcriptional activation during plant development. Biochim Biophys Acta 2011, 1809:567-576.
16. Wang $X$, Wang H, Wang J, Sun $R$, Wu J, Liu S, Bai Y, Mun JH, Bancroft I, Cheng F, Huang S, Li X, Hua W, Wang J, Wang X, Freeling M, Pires JC, Paterson AH, Chalhoub B, Wang B, Hayward A, Sharpe AG, Park BS, Weisshaar B, Liu B, Li B, Liu B, Tong C, Song C, Duran C, Peng C, Geng C, Koh C, Lin C, Edwards D, Mu D, Shen D, Soumpourou E, Li F, Fraser F, Conant G, Lassalle G, King GJ, Bonnema G, Tang H, Wang H, Belcram H, Zhou H, Hirakawa H, Abe H, Guo H, Wang H, Jin H, Parkin IA, Batley J, Kim JS, Just J, Li J, Xu J, Deng J, Kim JA, Li J, Yu J, Meng J, Wang J, Min J, Poulain J, Wang J, Hatakeyama K, Wu K, Wang L, Fang L, Trick M, Links MG, Zhao M, Jin M, Ramchiary N, Drou N, Berkman PJ, Cai Q, Huang Q, Li R, Tabata S, Cheng S, Zhang S, Zhang S, Huang S, Sato S, Sun S, Kwon SJ, Choi SR, Lee TH, Fan W, Zhao X, Tan X, Xu X, Wang Y, Qiu Y, Yin Y, Li Y, Du Y, Liao Y, Lim Y, Narusaka Y, Wang Y, Wang Z, Li Z, Wang Z, Xiong Z, Zhang Z: The genome of the mesopolyploid crop species Brassica rapa. Nat Genet 2011, 43:1035-1039.

17. Allis $C D$, Berger $S L$, Cote J, Dent $S$, Jenuwien $T$, Kouzarides $T$, Pillus $L$, Reinberg D, Shi Y, Shiekhattar R, Shilatifard A, Workman J, Zhang Y: New nomenclature for chromatin-modifying enzymes. Cell 2007, 131(4):633-636.

18. Briggs SD, Bryk M, Strahl BD, Cheung WL, Davie JK, Dent SY, Winston F, Allis CD: Histone $\mathrm{H} 3$ lysine 4 methylation is mediated by set1 and required for cell growth and rDNA silencing in Saccharomyces cerevisiae. Genes Dev 2001, 15:3286-3295.

19. Strahl BD, Grant PA, Briggs SD, Sun ZW, Bone JR, Caldwell JA, Mollah S, Cook RG, Shabanowitz J, Hunt DF, Allis CD: Set2 is a nucleosomal histone $\mathrm{H} 3$-selective methyltransferase that mediates transcriptional repression. Mol Cell Biol 2002, 22:1298-1306.

20. Ng DW, Wang T, Chandrasekharan MB, Aramayo R, Kertbundit S, Hall TC: Plant SET domain-containing proteins: structure, function and regulation. Biochim Biophys Acta 2007, 1769:316-329.

21. Johnson LM, Bostick M, Zhang X, Kraft E, Henderson I, Callis J, Jacobsen SE: The SRA methyl-cytosine-binding domain links DNA and histone methylation. Curr Biol 2007, 17:379-384.

22. Rajakumara $E$, Law JA, Simanshu DK, Voigt $P$, Johnson $L M$, Reinberg $D$, Patel DJ, Jacobsen SE: A dual flip-out mechanism for $5 \mathrm{mC}$ recognition by the Arabidopsis SUVH5 SRA domain and its impact on DNA methylation and H3K9 dimethylation in vivo. Genes Dev 2011, 25:137-152.

23. Rea S, Eisenhaber F, O'Carroll D, Strahl BD, Sun ZW, Schmid M, Opravil S, Mechtler $\mathrm{K}$, Ponting $C P$, Allis $C D$, Jenuwein $T$ : Regulation of chromatin structure by site-specific histone $\mathrm{H} 3$ methyltransferases. Nature 2000, 406:593-599.

24. Tachibana M, Sugimoto K, Fukushima T, Shinkai Y: Set domain-containing protein, G9a, is a novel lysine-preferring mammalian histone methyltransferase with hyperactivity and specific selectivity to lysines 9 and 27 of histone H3. J Biol Chem 2001, 276:25309-25317.

25. Malagnac F, Bartee L, Bender J: An Arabidopsis SET domain protein required for maintenance but not establishment of DNA methylation. EMBO J 2002, 21:6842-6852.

26. Ebbs ML, Bartee $L$, Bender J: H3 lysine 9 methylation is maintained on a transcribed inverted repeat by combined action of SUVH6 and SUVH4 methyltransferases. Mol Cell Biol 2005, 25:10507-10515.

27. Ebbs ML, Bender J: Locus-specific control of DNA methylation by the Arabidopsis SUVH5 histone methyltransferase. Plant Cell 2006, 18:1166-1176.

28. Naumann K, Fischer A, Hofmann I, Krauss V, Phalke S, Irmler K, Hause G, Aurich AC, Dorn R, Jenuwein T, Reuter G: Pivotal role of AtSUVH2 in heterochromatic histone methylation and gene silencing in Arabidopsis. EMBO J 2005, 24:1418-1429.

29. Ding Y, Wang X, Su L, Zhai J, Cao S, Zhang D, Liu C, Bi Y, Qian Q, Cheng Z, Chu C, Cao X: SDG714, a histone H3K9 methyltransferase, is involved in Tos17 DNA methylation and transposition in rice. Plant Cell 2007, 19:9-22.

30. Ding B, Zhu Y, Bu ZY, Shen WH, Yu Y, Dong AW: SDG714 regulates specific gene expression and consequently affects plant growth via $\mathrm{H} 3 \mathrm{~K} 9$ dimethylation. J Integr Plant Biol 2010, 52:420-430.

31. Qin FJ, Sun QW, Huang LM, Chen XS, Zhou DX: Rice SUVH histone methyltransferase genes display specific functions in chromatin modification and retrotransposon repression. Mol Plant 2010, 3:773-782.

32. Veiseth SV, Rahman MA, Yap KL, Fischer A, Egge-Jacobsen W, Reuter G, Zhou MM, Aalen RB, Thorstensen T: The SUVR4 histone lysine methyltransferase binds ubiquitin and converts $\mathrm{H} 3 \mathrm{~K} 9 \mathrm{me} 1$ to H3K9me3 on transposon chromatin in Arabidopsis. PLoS Genet 2011, 7:e1001325. 
33. Krichevsky A, Gutgarts H, Kozlovsky SV, Tzfira T, Sutton A, Sternglanz R, Mandel G, Citovsky V: C2H2 zinc finger-SET histone methyltransferase is a plant-specific chromatin modifier. Dev Biol 2007, 303:259-269.

34. Qiu C, Sawada K, Zhang X, Cheng X: The PWWP domain of mammalian DNA methyltransferase Dnmt3b defines a new family of DNA-binding folds. Nat Struct Biol 2002, 9:217-224.

35. Vezzoli A, Bonadies N, Allen MD, Freund SM, Santiveri CM, Kvinlaug BT, Huntly BJ, Gottgens B, Bycroft M: Molecular basis of histone H3K36me3 recognition by the PWWP domain of Brpf1. Nat Struct Mol Biol 2010, 17:617-619.

36. Aasland R, Gibson TJ, Stewart AF: The PHD finger: implications for chromatin-mediated transcriptional regulation. Trends Biochem Sci 1995, 20:56-59.

37. Yun $M$, Wu J, Workman JL, Li B: Readers of histone modifications. Cell Res 2011, 21:564-578.

38. Schultz J, Copley RR, Doerks T, Ponting CP, Bork P: SMART: a web-based tool for the study of genetically mobile domains. Nucleic Acids Res 2000, 28:231-234.

39. Kofler MM, Freund C: The GYF domain. FEBS J 2006, 273:245-256.

40. Alvarez-Venegas R, Pien S, Sadder M, Witmer X, Grossniklaus U, Avramova Z: ATX-1, an Arabidopsis homolog of trithorax, activates flower homeotic genes. Curr Biol 2003, 13:627-637.

41. Saleh A, Alvarez-Venegas R, Yilmaz M, Le O, Hou G, Sadder M, Al-Abdallat A, Xia Y, Lu G, Ladunga I, Avramova Z: The highly similar Arabidopsis homologs of trithorax ATX1 and ATX2 encode proteins with divergent biochemical functions. Plant Cell 2008, 20:568-579.

42. Pien S, Fleury D, Mylne JS, Crevillen P, Inze D, Avramova Z, Dean C, Grossniklaus U: ARABIDOPSIS TRITHORAX1 dynamically regulates FLOWERING LOCUS C activation via histone 3 lysine 4 trimethylation. Plant Cell 2008, 20:580-588.

43. Berr A, Xu L, Gao J, Cognat V, Steinmetz A, Dong A, Shen WH: SET DOMAIN GROUP25 encodes a histone methyltransferase and is involved in FLOWERING LOCUS C activation and repression of flowering. Plant Physiol 2009, 151:1476-1485.

44. Tamada Y, Yun JY, Woo SC, Amasino RM: ARABIDOPSIS TRITHORAXRELATED7 is required for methylation of lysine 4 of histone $\mathrm{H} 3$ and for transcriptional activation of FLOWERING LOCUS C. Plant Cell 2009, 21:3257-3269.

45. Doerks T, Copley RR, Schultz J, Ponting CP, Bork P: Systematic identification of novel protein domain families associated with nuclear functions. Genome Res 2002, 12:47-56.

46. Hoppmann V, Thorstensen T, Kristiansen PE, Veiseth SV, Rahman MA, Finne K, Aalen RB, Aasland R: The CW domain, a new histone recognition module in chromatin proteins. EMBO J 2011, 30:1939-1952.

47. Xu L, Zhao Z, Dong A, Soubigou-Taconnat L, Renou JP, Steinmetz A, Shen WH: Di- and tri- but not monomethylation on histone H3 lysine 36 marks active transcription of genes involved in flowering time regulation and other processes in Arabidopsis thaliana. Mol Cell Biol 2008, 28:1348-1360.

48. Kim SY, He Y, Jacob Y, Noh YS, Michaels S, Amasino R: Establishment of the vernalization-responsive, winter-annual habit in Arabidopsis requires a putative histone H3 methyl transferase. Plant Cell 2005, 17:3301-3310.

49. Zhao Z, Yu Y, Meyer D, Wu C, Shen WH: Prevention of early flowering by expression of FLOWERING LOCUS C requires methylation of histone $\mathrm{H} 3$ K36. Nat Cell Biol 2005, 7:1256-1260.

50. Dong G, Ma DP, Li J: The histone methyltransferase SDG8 regulates shoot branching in Arabidopsis. Biochem Biophys Res Commun 2008, 373:659-664.

51. Cazzonelli Cl, Cuttriss AJ, Cossetto SB, Pye W, Crisp P, Whelan J, Finnegan EJ, Turnbull C, Pogson BJ: Regulation of carotenoid composition and shoot branching in Arabidopsis by a chromatin modifying histone methyltransferase, SDG8. Plant Cell 2009, 21:39-53.

52. Grini PE, Thorstensen T, Alm V, Vizcay-Barrena G, Windju SS, Jorstad TS, Wilson ZA, Aalen RB: The ASH1 HOMOLOG 2 (ASHH2) histone H3 methyltransferase is required for ovule and anther development in Arabidopsis. PLoS One 2009, 4:e7817.

53. Berr A, McCallum EJ, Alioua A, Heintz D, Heitz T, Shen WH: Arabidopsis histone methyltransferase SET DOMAIN GROUP8 mediates induction of the jasmonate/ethylene pathway genes in plant defense response to necrotrophic fungi. Plant Physiol 2010, 154:1403-1414.
54. Palma K, Thorgrimsen S, Malinovsky FG, Fiil BK, Nielsen HB, Brodersen $P$, Hofius D, Petersen M, Mundy J: Autoimmunity in Arabidopsis acd11 is mediated by epigenetic regulation of an immune receptor. PLOS Pathog 2010, 6(10):e1001137.

55. Cartagena JA, Matsunaga S, Seki M, Kurihara D, Yokoyama M, Shinozaki K, Fujimoto S, Azumi Y, Uchiyama S, Fukui K: The Arabidopsis SDG4 contributes to the regulation of pollen tube growth by methylation of histone H3 lysines 4 and 36 in mature pollen. Dev Biol 2008, 315:355-368.

56. Thorstensen T, Grini PE, Mercy IS, Alm V, Erdal S, Aasland R, Aalen RB: The Arabidopsis SET-domain protein ASHR3 is involved in stamen development and interacts with the bHLH transcription factor ABORTED MICROSPORES (AMS). Plant Mol Biol 2008, 66:47-59.

57. Zheng B, Chen X: Dynamics of histone $\mathrm{H} 3$ lysine 27 trimethylation in plant development. Curr Opin Plant Biol 2011, 14:123-129.

58. Zhang D, Martyniuk CJ, Trudeau VL: SANTA domain: a novel conserved protein module in Eukaryota with potential involvement in chromatin regulation. Bioinformatics 2006, 22:2459-2462.

59. Jacob Y, Feng S, LeBlanc CA, Bernatavichute $Y$, Stroud H, Cokus S, Johnson LM, Pellegrini M, Jacobsen SE, Michaels SD: ATXR5 and ATXR6 are H3K27 monomethyltransferases required for chromatin structure and gene silencing. Nat Struct Mol Biol 2009, 16:763-768.

60. Jacob Y, Stroud H, Leblanc C, Feng S, Zhuo L, Caro E, Hassel C, Gutierrez C, Michaels SD, Jacobsen SE: Regulation of heterochromatic DNA replication by histone H3 lysine 27 methyltransferases. Nature 2010, 466:987-991.

61. Berr A, McCallum EJ, Menard R, Meyer D, Fuchs J, Dong A, Shen WH: Arabidopsis SET DOMAIN GROUP2 is required for H3K4 trimethylation and is crucial for both sporophyte and gametophyte development. Plant Cell 2010, 22:3232-3248.

62. Guo L, Yu Y, Law JA, Zhang X: SET DOMAIN GROUP2 is the major histone $\mathrm{H} 3$ lysine 4 trimethyltransferase in Arabidopsis. Proc Natl Acad Sci USA 2010, 107:18557-18562

63. Aquea F, Vega A, Timmermann T, Poupin MJ, Arce-Johnson P: Genomewide analysis of the SET DOMAIN GROUP family in Grapevine. Plant Cell Rep 2011, 30:1087-1097.

64. Price CA: Commission on plant gene nomenclature. Plant Mol Biol Rep 1993, 11:273-274.

65. Fischer A, Hofmann I, Naumann K, Reuter G: Heterochromatin proteins and the control of heterochromatic gene silencing in Arabidopsis. J Plant Physiol 2006, 163:358-368.

66. Lynch M, Force $A$ : The probability of duplicate gene preservation by subfunctionalization. Genetics 2000, 154:459-473.

67. Cannon SB, Mitra A, Baumgarten A, Young ND, May G: The roles of segmental and tandem gene duplication in the evolution of large gene families in Arabidopsis thaliana. BMC Plant Biol 2004, 4:10.

68. Luo M, Bilodeau P, Koltunow A, Dennis ES, Peacock WJ, Chaudhury AM: Genes controlling fertilization-independent seed development in Arabidopsis thaliana. Proc Natl Acad Sci USA 1999, 96:296-301.

69. Thompson JD, Higgins DG, Gibson TJ: CLUSTAL W: improving the sensitivity of progressive multiple sequence alignment through sequence weighting, position-specific gap penalties and weight matrix choice. Nucleic Acids Res 1994, 22:4673-4680.

70. Tamura K, Dudley J, Nei M, Kumar S: MEGA4: Molecular Evolutionary Genetics Analysis (MEGA) software version 4.0. Mol Biol Evol 2007, 24:1596-1599.

doi:10.1186/1471-2229-11-175

Cite this article as: Huang et al:: Phylogenetic analysis and classification of the Brassica rapa SET-domain protein family. BMC Plant Biology 2011 $11: 175$. 